\title{
Rotating Dilaton Black Strings Coupled to Exponential Nonlinear Electrodynamics
}

\author{
Ahmad Sheykhi ${ }^{1,2}$ \\ ${ }^{1}$ Physics Department and Biruni Observatory, College of Sciences, Shiraz University, Shiraz 71454, Iran \\ ${ }^{2}$ Research Institute for Astronomy and Astrophysics of Maragha (RIAAM), P.O. Box 55134-441, Maragha, Iran \\ Correspondence should be addressed to Ahmad Sheykhi; asheykhi@shirazu.ac.ir
}

Received 2 April 2014; Revised 21 September 2014; Accepted 2 October 2014; Published 20 October 2014

Academic Editor: Shi-Hai Dong

Copyright (c) 2014 Ahmad Sheykhi. This is an open access article distributed under the Creative Commons Attribution License, which permits unrestricted use, distribution, and reproduction in any medium, provided the original work is properly cited. The publication of this article was funded by $\mathrm{SCOAP}^{3}$.

\begin{abstract}
We construct a new class of charged rotating black string solutions coupled to dilaton and exponential nonlinear electrodynamic fields with cylindrical or toroidal horizons in the presence of a Liouville-type potential for the dilaton field. Due to the presence of the dilaton field, the asymptotic behaviors of these solutions are neither flat nor (A)dS. We analyze the physical properties of the solutions in detail. We compute the conserved and thermodynamic quantities of the solutions and verify the first law of thermodynamics on the black string horizon. When the nonlinear parameter $\beta^{2}$ goes to infinity, our results reduce to those of black string solutions in Einstein-Maxwell-dilaton gravity.
\end{abstract}

\section{Introduction}

The field equations of Einstein theory of general relativity have nonlinear nature. When these field equations are coupled to the linear Maxwell field, they admit the wellknown charged static and spherically symmetric ReissnerNordström (RN) black hole solution. However, various problems of divergence appear in Maxwell theory. For example, the self-energy of charged point particles diverges at its location. In order to overcome these difficulties the linear Maxwell theory was extended to the nonlinear electrodynamics. Up to now, three types of nonlinear Lagrangian for electrodynamics have proposed. Historically, the first nonlinear Lagrangian for the electromagnetics was proposed by Born and Infeld (BI) in 1934 [1]. The second type of nonlinear Lagrangian which has the logarithmic (LN) form was introduced by Soleng [2], and recently the exponential nonlinear (EN) Lagrangian for the electrodynamics was suggested by Hendi [3-5] as the third type of nonlinear electrodynamics. All of these Lagrangians have a nonlinear parameter $\beta$, for which their series expansion for large $\beta$ can be written as

$$
L_{\mathrm{BI}}=L_{\mathrm{LN}}=L_{\mathrm{EN}}=-F^{2}+\frac{F^{4}}{8 \beta^{2}}+O\left(\frac{1}{\beta^{4}}\right),
$$

where $\beta$ has dimension of mass, $F^{2}=F_{\mu \nu} F^{\mu \nu}$, where $F_{\mu \nu}$ is the electromagnetic field tensor. When $\beta^{2} \rightarrow \infty$, all of these Lagrangians recover the well-known Maxwell Lagrangian. A new class of regular black holes which is supported by a nonlinear electrodynamic source were studied in [6]. This solution corresponds to charged black holes, having the metric and electric field regular everywhere [6].

There are several motivations for studying nonlinear electrodynamics. First, nonlinear field theories are of interest to different branches of mathematical physics because most physical systems are inherently nonlinear in nature. Nonlinear action of Abelian gauge theories has been considered in the context of superstring theory [7-14]. Second, modifying the linear Maxwell theory to the nonlinear electrodynamics 
theory can eliminate the problem of divergency in electromagnetic field and leads to finite self-energy and finite electric potential at the origin. Third, it is also remarkable that BItype theories are singled out among the classes of nonlinear theories by their special properties such as the absence of shock waves, birefringence phenomena $[15,16]$, and enjoying an electric-magnetic duality [17]. From the thermodynamics point of view, nonlinear electrodynamics also leads to some interesting properties for the black hole systems and has been widely studied in the literatures. In addition, from the point of view of AdS/CFT correspondence in hydrodynamic models, it has been shown that, unlike gravitational correction, higher-derivative terms for Abelian fields in the form of NLED do not affect the ratio of shear viscosity over entropy density [18-21]. In addition, in applications of the AdS/CFT correspondence to superconductivity, NLED theories make crucial effects on the condensation as well as the critical temperature of the superconductor and its energy gap [22, 23]. Furthermore, it has been shown that the effects of NLED become important when we investigate super-strongly magnetized compact objects, such as pulsars, neutron stars, magnetars, and strange quark magnetars [24, 25].

On the other side, a scalar field called dilaton appears in the low energy limit of string theory. Various aspects of black hole spacetimes in the context of dilaton gravity have been studied in the literatures. In the absence of dilaton potential, exact solutions of charged dilaton black holes with flat asymptotic have been constructed in [26-32]. When the Liouville-type dilaton potential, which is regarded as the generalization of the cosmological constant, is taken into account, the asymptotic behavior of the solutions is changed to be neither asymptotically flat nor (anti-)de Sitter [(A)dS] [33-35]. In the presence of one or two Liouville-type potentials, black hole spacetimes which are neither asymptotically flat nor (A)dS have been explored by many authors (see, e.g., [36-45]). The BI action coupled to the dilaton field appears in the low energy limit of open superstring theory [7-11]. Black hole solutions in the context of Einstein-Born-Infelddilaton (EBId) gravity in three [46] and four dimensions have been investigated [47-52]. Thermodynamics of $(n+$ 1)-dimensional EBId solutions with flat [53] and curved horizons have also been explored [54, 55]. A class of spinning magnetic brane in $(n+1)$-dimensional EBId gravity with Liouville type potential which produces a longitudinal nonlinear electromagnetic field was presented in [56]. In the scalar-tensor theories of gravity, black holes solution coupled to BI nonlinear electrodynamics have also been studied in $[57,58]$. Recently, the studies were also generalized to EN electrodynamics coupled to the dilaton field (ENd). In this regard, a new class of static and spherically symmetric solutions in four dimensional-ENd gravity was constructed in [59].

In this paper, we would like to extend the study on ENd theory by constructing a new class of rotating dilaton black string solutions with cylindrical or toroidal horizons in the presence of EN electromagnetic field. We will present the suitable counterterm which removes the divergences of the action. We also study thermodynamics of the obtained black string solutions and verify the first law of thermodynamics on the horizon. We finish our paper with conclusions in the last section.

\section{Field Equations and Black String Solutions}

We start with the four-dimensional action in which gravity is coupled to the dilaton and EN electrodynamic fields,

$$
\begin{aligned}
I_{G}= & -\frac{1}{16 \pi} \int_{\mathscr{M}} d^{4} x \sqrt{-g}\left(R-2 \partial_{\mu} \Phi \partial^{\mu} \Phi-V(\Phi)+L(F, \Phi)\right) \\
& -\frac{1}{8 \pi} \int_{\partial \mathscr{M}} d^{3} x \sqrt{-h} \Theta,
\end{aligned}
$$

where $R$ is the Ricci scalar curvature, $\Phi$ is the dilaton field, and $V(\Phi)$ is a potential for $\Phi$. We choose the Lagrangian of the EN electrodynamics coupled to the dilaton field (ENd), $L(F, \Phi)$, as

$$
L(F, \Phi)=4 \beta^{2} e^{2 \alpha \Phi}\left[\exp \left(-\frac{e^{-4 \alpha \Phi} F^{2}}{4 \beta^{2}}\right)-1\right],
$$

where $\alpha$ is a constant determining the strength of coupling of the scalar and electromagnetic fields, $F_{\mu \nu}=\partial_{\mu} A_{v}-$ $\partial_{\nu} A_{\mu}$ is the electromagnetic field tensor, and $A_{\mu}$ is the electromagnetic potential. The last term in (2) is the GibbonsHawking surface term, which guaranties the variation of the action to be well-defined. The factor $\Theta$ is the trace of the extrinsic curvature for the boundary $\partial \mathscr{M}$ and $h$ is the induced metric on the boundary. Before we proceed further, let us give some motivations for introducing the Lagrangian (3). The first reason for taking this kind of Lagrangian originates from the fact that for large value of nonlinear parameter $\beta$, it leads to the well-known Maxwell-dilaton Lagrangian [36], exactly like BI lagrangian coupled to the dilaton field in the limit of large $\beta$,

$$
\left.L(F, \Phi)\right|_{\operatorname{large} \beta}=-e^{-2 \alpha \Phi} F^{2}+O\left(\frac{1}{\beta^{2}}\right) .
$$

This strongly implies that the nonlinear electrodynamics Lagrangian (3) proposed here is a toy model and also deserves further study. The advantages of the Lagrangian (3) compared to the BI nonlinear Lagrangian is that while the self-energy of charged point particle in BI theory has finite value near the origin, it becomes very large as $r \rightarrow 0$ for (3); however, the singularity is much weaker than Maxwell theory [3-5]. This means that the proposed Lagrangian in this paper is more realistic than BI Lagrangian and has a behavior between BI and Maxwell theory. Besides, as we mentioned already, the BI-type electrodynamics naturally arises in the low energy limit of the open string theory [7-14]. However, in string theory it may appear as a series expansion and the freedom of choosing the coefficients of the series expansion allows us to collect the terms and write them in a different compact form such as BI, EN, or LN electrodynamics. The reason is that all of these three Lagrangians have the similar expansion. In addition, in the absence of the dilaton field $(\alpha=0), L(F, \Phi)$ reduces to the Lagrangian of EN electrodynamics which was 
already studied in [5] and the corresponding black string solutions were investigated [5]. The lagrangian proposed in (3) is the quite one generalization of lagrangian [5] to include the dilaton scalar field.

For latter convenience we write the lagrangian as

$$
L(F, \Phi)=4 \beta^{2} e^{2 \alpha \Phi} \mathscr{L}(Y),
$$

where we have defined

$$
\begin{aligned}
\mathscr{L}(Y) & =\exp (-Y)-1, \\
Y & =\frac{e^{-4 \alpha \Phi} F^{2}}{4 \beta^{2}} .
\end{aligned}
$$

In this paper, we consider action (2) with a Liouville type potential,

$$
V(\Phi)=2 \Lambda e^{2 \alpha \Phi}
$$

where $\Lambda$ is a constant which may be referred to as the cosmological constant, since in the absence of the dilaton field $(\Phi=0)$ the action (2) reduces to the action of EN electrodynamics in Einstein gravity in the presence of cosmological constant [5]. The equations of motion can be obtained by varying the action (2) with respect to the gravitational field $g_{\mu \nu}$, the dilaton field $\Phi$, and the gauge field $A_{\mu}$ which yields the following field equations:

$$
\begin{aligned}
& \mathscr{R}_{\mu \nu}= 2 \partial_{\mu} \Phi \partial_{\nu} \Phi+\frac{1}{2} g_{\mu \nu} V(\Phi)-2 e^{-2 \alpha \Phi} \\
& \times \partial_{Y} \mathscr{L}(Y) F_{\mu \eta} F_{\nu}^{\eta}+2 \beta^{2} e^{2 \alpha \Phi} \\
& \times\left[2 Y \partial_{Y} \mathscr{L}(Y)-\mathscr{L}(Y)\right] g_{\mu \nu}, \\
& \nabla^{2} \Phi=\frac{1}{4} \frac{\partial V}{\partial \Phi}+2 \alpha \beta^{2} e^{2 \alpha \Phi}\left[2 Y \partial_{Y} \mathscr{L}(Y)-\mathscr{L}(Y)\right], \\
& \nabla_{\mu}\left(e^{-2 \alpha \Phi} \partial_{Y} \mathscr{L}(Y) F^{\mu \nu}\right)=0 .
\end{aligned}
$$

In case of linear electrodynamics we have $\mathscr{L}(Y)=-Y$, and the system of (8)-(10) reduce to the well-known equations of Einstein-Maxwell-dilaton (EMd) gravity [36].

Our aim here is to construct charged rotating black string solutions of the field equations (8)-(10) and investigate their properties. The metric of four-dimensional rotating solution with cylindrical or toroidal horizons can be written as $[60,61]$

$$
\begin{aligned}
d s^{2}= & -f(r)(\Xi d t-a d \phi)^{2}+r^{2} R^{2}(r)\left(\frac{a}{l^{2}} d t-\Xi d \phi\right)^{2} \\
& +\frac{d r^{2}}{f(r)}+\frac{r^{2}}{l^{2}} R^{2}(r) d z^{2},
\end{aligned}
$$

where $\Xi=\sqrt{1+a^{2} / l^{2}}$ and $a$ is the rotation parameter as we will see later. The functions $f(r)$ and $R(r)$ should be determined and $l$ has the dimension of length which is related to the constant $\Lambda$ by the relation $l^{2}=-3 / \Lambda$. The two dimensional space, $t=$ constant and $r=$ constant, can be (i) the flat torus model $T^{2}$ with topology $S^{1} \times S^{1}$ and $0 \leq \phi<2 \pi$,
$0 \leq z<2 \pi l$, (ii) the standard cylindrical model with topology $R \times S^{1}$ and $0 \leq \phi<2 \pi,-\infty<z<\infty$, and (iii) the infinite plane $R^{2}$ with $-\infty<\phi<\infty$ and $-\infty<z<\infty$. We will focus upon (i) and (ii).

The modified Maxwell equation (10) can be integrated immediately to give

$$
\begin{aligned}
& F_{t r}=\frac{q \Xi e^{2 \alpha \Phi}}{r^{2} R^{2}(r)} \exp \left[-\frac{1}{2} L_{W}\left(\frac{q^{2}}{\beta^{2} r^{4} R^{4}(r)}\right)\right], \\
& F_{\phi r}=-\frac{a}{\Xi} F_{t r},
\end{aligned}
$$

where $q$ is an integration constant which is related to the electric charge of the black string, and $L_{W}(x)=\operatorname{Lambert} W(x)$ is the Lambert function which satisfies $[62,63]$

$$
L_{W}(x) e^{L_{W}(x)}=x,
$$

and it has the following series expansion:

$$
L_{W}(x)=x-x^{2}+\frac{3}{2} x^{3}-\frac{8}{3} x^{4}+\cdots .
$$

Clearly, series (15) converges for $|x|<1$. The expansion of (12) for large $\beta$ (or large $r$ ) is given by

$$
F_{t r}=\frac{q \Xi e^{2 \alpha \Phi}}{(r R)^{2}}-\frac{\Xi}{2 \beta^{2}} \frac{q^{3} e^{2 \alpha \Phi}}{(r R)^{6}}+\frac{5 \Xi}{8 \beta^{4}} \frac{q^{5} e^{2 \alpha \Phi}}{(r R)^{10}}+O\left(\frac{1}{\beta^{6}}\right) .
$$

In the absence of the dilaton field ( $\alpha=0, R(r)=1)$, (12) reduces to

$$
F_{t r}=\frac{q \Xi}{r^{2}} \exp \left[-\frac{1}{2} L_{W}\left(\frac{q^{2}}{\beta^{2} r^{4}}\right)\right],
$$

while its expansion (16) reduces to

$$
F_{t r}=\frac{q \Xi}{r^{2}}-\frac{q^{3} \Xi}{2 \beta^{2} r^{6}}+\frac{5 q^{5} \Xi}{8 \beta^{4} r^{10}}+O\left(\frac{1}{\beta^{6}}\right),
$$

Now, we want to solve the system of (8) and (9) for three unknown functions $f(r), R(r)$, and $\Phi(r)$. To this end, we make the suitable ansatz [41]

$$
R(r)=e^{\alpha \Phi} .
$$

Using (19), the electromagnetic fields (12) and (13) and metric (11), after some mathematic calculations, we can show that (8) and (9) have solution of the form

$$
\begin{aligned}
f(r)= & \frac{\left(\Lambda+2 \beta^{2}\right)\left(\alpha^{2}+1\right)^{2} b^{\gamma}}{\alpha^{2}-3} r^{2-\gamma}-\frac{m}{r^{1-\gamma}} \\
& -\frac{2 \beta q}{r^{1-\gamma}}\left(\alpha^{2}+1\right) b^{\gamma} \\
& \times \int r^{-\gamma}\left(\sqrt{L_{W}(\eta)}-\frac{1}{\sqrt{L_{W}(\eta)}}\right) d r, \\
\Phi(r)= & \frac{\alpha}{\alpha^{2}+1} \ln \left(\frac{b}{r}\right),
\end{aligned}
$$


where $\gamma=2 \alpha^{2} /\left(1+\alpha^{2}\right)$ and

$$
\eta \equiv \frac{q^{2} r^{2 \gamma-4}}{\beta^{2} b^{2 \gamma}} .
$$

Here $b$ and $m$ are arbitrary constants. The integral can be done in terms of Gamma function and can be written in a compact form as

$$
\begin{aligned}
& f(r) \\
& =\frac{\left(\Lambda+2 \beta^{2}\right)\left(\alpha^{2}+1\right)^{2} b^{\gamma}}{\alpha^{2}-3} r^{2-\gamma}-\frac{m}{r^{1-\gamma}}+\frac{\beta q\left(\alpha^{2}+1\right)^{4}}{2\left(\alpha^{2}-1\right)^{2}} \\
& \times\left(\frac{\beta^{2} b^{2 \gamma}}{q^{2}}\right)^{(1-\gamma) /(2 \gamma-4)} r^{\gamma-1} \times\left(\frac{1-\alpha^{2}}{4}\right)^{(2 \gamma-3) /(2 \gamma-4)} \\
& \times\left\{-4(\gamma-2)^{2}\left[\Gamma\left(\frac{\alpha^{2}+5}{4}, \frac{1-\alpha^{2}}{4} L_{W}(\eta)\right)\right.\right. \\
& \left.\left.\times\left[\Gamma\left(\frac{\alpha^{2}-3}{4}, \frac{1-\alpha^{2}}{4} L_{W}(\eta)\right)-\Gamma\left(\frac{\alpha^{2}+5}{4}\right)\right]+(\gamma-1)^{2}\right)\right]
\end{aligned}
$$

where $\Gamma(a, z)$ and $\Gamma(a)$ are Gamma functions which satisfy

$$
\Gamma(a, z)=\Gamma(a)-\frac{z^{a}}{a} F(a, 1+a,-z) .
$$

Here $F(a, b, z)$ is the hypergeometric function [62, 63]. Using (23), solution (22) can also be reexpressed in terms of hypergeometric function,

$$
\begin{aligned}
f(r)= & \frac{\left(\Lambda+2 \beta^{2}\right)\left(\alpha^{2}+1\right)^{2} b^{\gamma}}{\alpha^{2}-3} r^{2-\gamma}-\frac{m}{r^{1-\gamma}} \\
& +2 \beta q\left(\alpha^{2}+1\right)^{2} r^{\gamma-1}\left(\frac{\beta^{2} b^{2 \gamma}}{q^{2}}\right)^{(1-\gamma) /(2 \gamma-4)} \\
& \times L_{W}^{(3-2 \gamma) /(2 \gamma-4)}(\eta) \\
& \times\left\{\frac{L_{W}^{2}(\eta)}{\alpha^{2}+5} F\left(\frac{\alpha^{2}+5}{4}, \frac{\alpha^{2}+9}{4}, \frac{\alpha^{2}-1}{4} L_{W}(\eta)\right)\right. \\
& \left.\quad-\frac{1}{\alpha^{2}-3} F\left(\frac{\alpha^{2}-3}{4}, \frac{\alpha^{2}+1}{4}, \frac{\alpha^{2}-1}{4} L_{W}(\eta)\right)\right\}
\end{aligned}
$$

In the absence of the dilaton field ( $\alpha=0=\gamma)$, our solution reduces to the black string solutions coupled to EN electrodynamics [5]. Using the fact that $L_{W}(x)$ has a convergent series expansion for $|x|<1$ as given in (15), we can expand (22) for large $\beta$ (or large $r$ ). We find

$$
\begin{aligned}
f(r)= & \frac{\Lambda\left(\alpha^{2}+1\right)^{2}}{\alpha^{2}-3} b^{\gamma} r^{2-\gamma}-\frac{m}{r^{1-\gamma}}+\frac{q^{2}\left(\alpha^{2}+1\right) b^{-\gamma}}{r^{2-\gamma}} \\
& -\frac{q^{4}\left(\alpha^{2}+1\right)^{2} b^{-3 \gamma}}{4 \beta^{2}\left(\alpha^{2}+5\right) r^{6-3 \gamma}}+O\left(\frac{1}{\beta^{4}}\right) .
\end{aligned}
$$

When $\beta \rightarrow \infty$, it restores charged rotating black string solutions of EMd gravity [41], as one expects. The $1 / \beta^{2}$ term in the right hand side of the above expression is the leading EN electrodynamic correction to the black string with dilaton field. In the absence of the dilaton field $(\alpha=0=\gamma)$, solution (25) reduces to black string solutions in Einstein gravity (as $\beta \rightarrow \infty)[60,61]$

$$
f(r)=\frac{r^{2}}{l^{2}}-\frac{m}{r}+\frac{q^{2}}{r^{2}} .
$$

Properties of the Solutions. Next, we study the geometry of spacetime. For this purpose, we first seek for the curvature singularities in the presence of dilaton and nonlinear electrodynamic fields. It is a matter of calculation to show that the Ricci scalar and the Kretschmann invariant behave as black

$$
\begin{gathered}
\lim _{r \rightarrow 0^{+}} R=\infty, \\
\lim _{r \rightarrow 0^{+}} R_{\mu \nu \rho \sigma} R^{\mu \nu \rho \sigma}=\infty,
\end{gathered}
$$

which indicate that there is an essential singularity at $r=$ 0 . It is also easy to check that the spacetime is neither asymptotically flat nor (A)dS. This is due the presence of the Liouville-type dilaton potential. Indeed, it has been shown that no dilaton $\mathrm{dS}$ or AdS black hole solution exists with the presence of only one or two Liouville-type dilaton potentials [33-35].

One can obtain the temperature and angular momentum of the event horizon by analytic continuation of the metric. Setting $t \rightarrow i \tau$ and $a \rightarrow i a$ yields the Euclidean section of (11), whose regularity at $r=r_{+}$requires that we should identify $\tau \sim \tau+\beta_{+}$and $\phi \sim \phi+i \beta_{+} \Omega_{+}$, where $\beta_{+}$and $\Omega_{+}$are the inverse Hawking temperature and the angular velocities of the outer event horizon. It is easy to show that the angular velocity of the black string is [41]

$$
\Omega=\frac{a}{\Xi l^{2}}
$$

while the Hawking temperature is obtained as

$$
\begin{aligned}
T_{+}= & \frac{f^{\prime}\left(r_{+}\right)}{4 \pi \Xi}=-\frac{\left(\alpha^{2}+1\right)}{4 \pi \Xi} r_{+}^{1-\gamma} \\
& \times\left\{\left(\Lambda+2 \beta^{2}\right) b^{\gamma}-2 \beta q r_{+}^{\gamma-2}\right. \\
& \left.\times\left(\frac{1}{\sqrt{L_{W}\left(\eta_{+}\right)}}-\sqrt{L_{W}\left(\eta_{+}\right)}\right)\right\},
\end{aligned}
$$

where $\eta_{+}=\eta\left(r=r_{+}\right)$and we have used $f\left(r_{+}\right)=0$. The behavior of $T$ versus $r_{+}$is shown in Figure 1. From these figures we find out that, for large value of $r_{+}$, the temperature tends to a constant independent of the model parameters. 


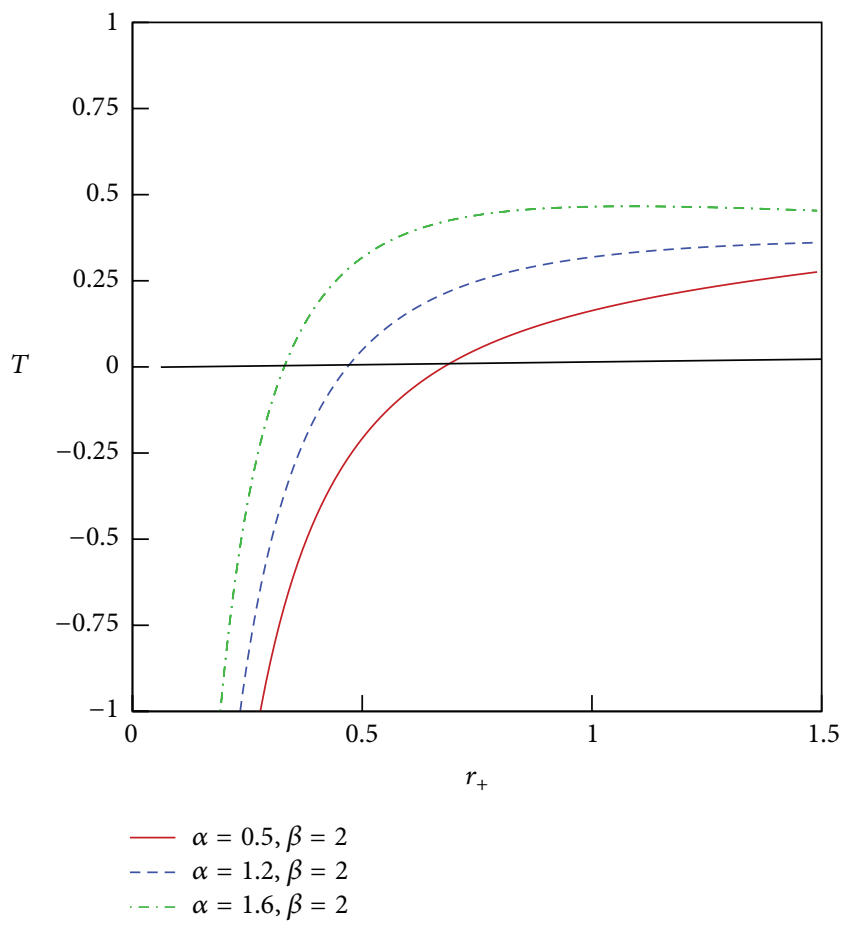

(a)

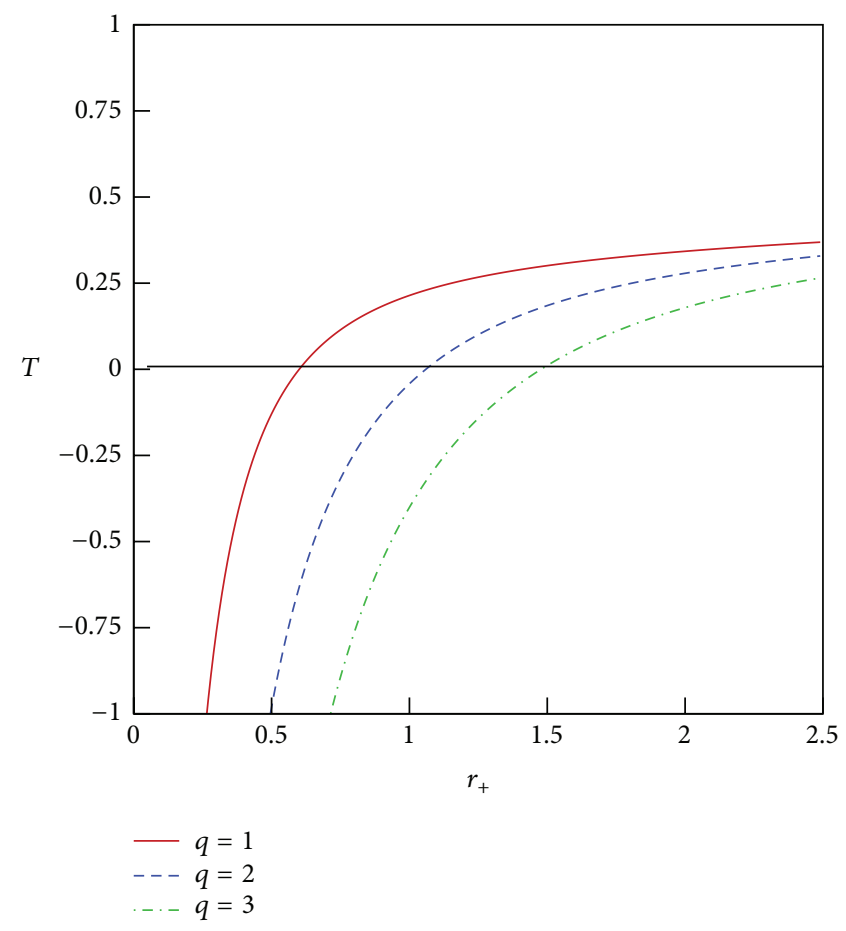

(b)

Figure 1: (a) shows the evolution of $T$ versus $r_{+}$for $q=l=b=1$ and $\Xi=1.25$. In (b) the behavior of $T$ is illustrated for $b=l=1, \Xi=1.25$, $\alpha=0.8$, and $\beta=2$.

On the other hand, for small values of $r_{+}$, the temperature may be negative $(T<0)$. Physically it is hard to accept negative temperature. Indeed, there is an extremal value for the event horizon radius $r_{\text {ext }}$, for which we have the black hole solution provided $r_{+} \geq r_{\text {ext }}$. In this case the temperature is not negative. In case of $r_{+}<r_{\text {ext }}$ we have no black hole solution. The temperature is zero and the horizon is degenerate for an extremal black hole provided $r_{+}=r_{\text {ext }}$. In this case $r_{\text {ext }}$ is the positive root of the following equation:

$$
\begin{aligned}
& \left(\Lambda+2 \beta^{2}\right) b^{\gamma} r_{\text {ext }}^{2-\gamma}-2 \beta q_{\text {ext }}\left(\frac{1}{\sqrt{L_{W}\left(\eta_{\text {ext }}\right)}}-\sqrt{L_{W}\left(\eta_{\text {ext }}\right)}\right) \\
& =0,
\end{aligned}
$$

where

$$
\eta_{\mathrm{ext}} \equiv \frac{q_{\mathrm{ext}}^{2} r_{\mathrm{ext}}^{2 \gamma-4}}{\beta^{2} b^{2 \gamma}}
$$

From Figure 1 we see that $r_{\text {ext }}$ decreases as $\alpha$ increases, while $r_{\text {ext }}$ increases with increasing $q$. Indeed, the metric of (11) and (22) can describe a nonlinear dilaton black hole with two horizons provided $r_{+}>r_{\text {ext }}$, an extreme ENd black hole in case of $r_{+}=r_{\text {ext }}$, and a naked singularity if $r_{+}<r_{\text {ext }}$. Note that in the limiting case where $\beta \rightarrow \infty$, expression (29) reduces to that of black string in EMd theory [41]

$$
T_{+}=-\frac{\Lambda\left(\alpha^{2}+1\right) b^{\gamma}}{4 \pi \Xi} r_{+}^{1-\gamma}-\frac{q^{2} b^{-\gamma}\left(\alpha^{2}+1\right)}{4 \pi \Xi} r_{+}^{\gamma-3}+O\left(\frac{1}{\beta^{2}}\right)
$$

From (25) we see that the solution is ill-defined for $\alpha=$ $\sqrt{3}$. Let us consider the case with $\alpha>\sqrt{3}$ and $\alpha<\sqrt{3}$, separately. When $\alpha>\sqrt{3}$, as $r$ goes to infinity the dominant term in (25) is the second term, and therefore the spacetime has a cosmological horizon for positive values of the mass parameter, despite the sign of the cosmological constant $\Lambda$. For $\alpha<\sqrt{3}$, as $r$ goes to infinity the dominant term in (25) is the first term, and therefore there exists a cosmological horizon for $\Lambda>0$, while there are no cosmological horizons if $\Lambda<0$. Indeed, in the latter case $(\alpha<\sqrt{3}$ and $\Lambda<0)$ the spacetimes associated with the solution (24) exhibit a variety of possible casual structures depending on the values of the metric parameters $\alpha, \beta, m, q$, and $\Lambda$. One can obtain the location of the horizons by finding the roots of $f(r)=0$. Unfortunately, due to the complexity of (24), it is not possible to find explicitly the location of the horizons. But, we can obtain some information by plotting $f(r)$ as a function of $r$ for different parameters (see Figure 2). These figures show that the obtained solutions may represent a black hole with two horizons, an extreme black hole or a naked singularity 


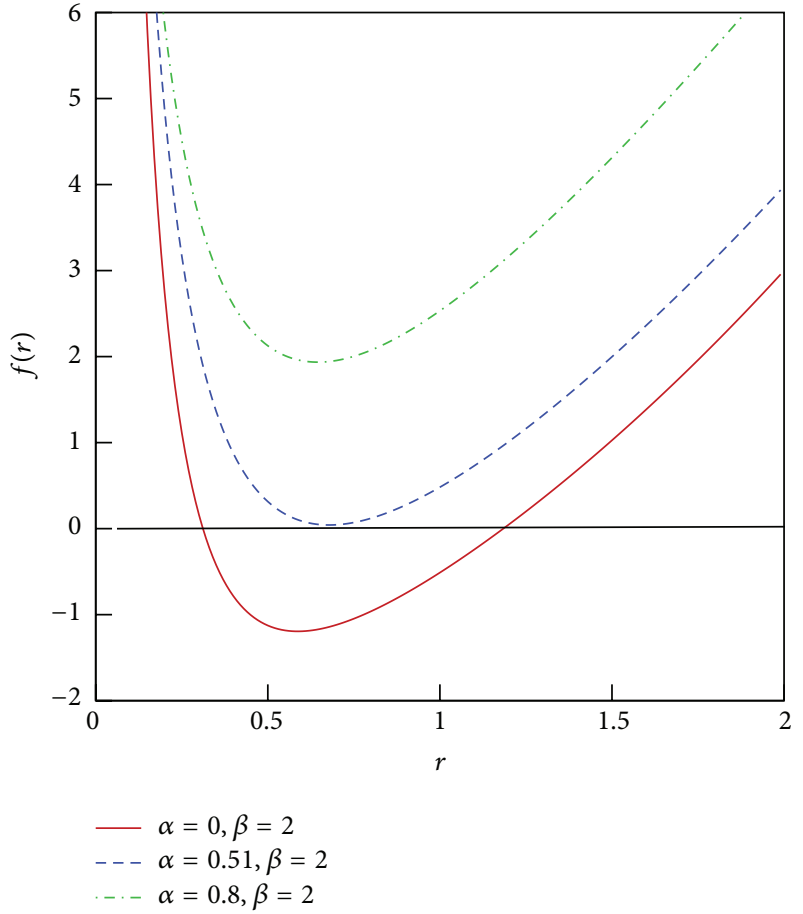

(a)

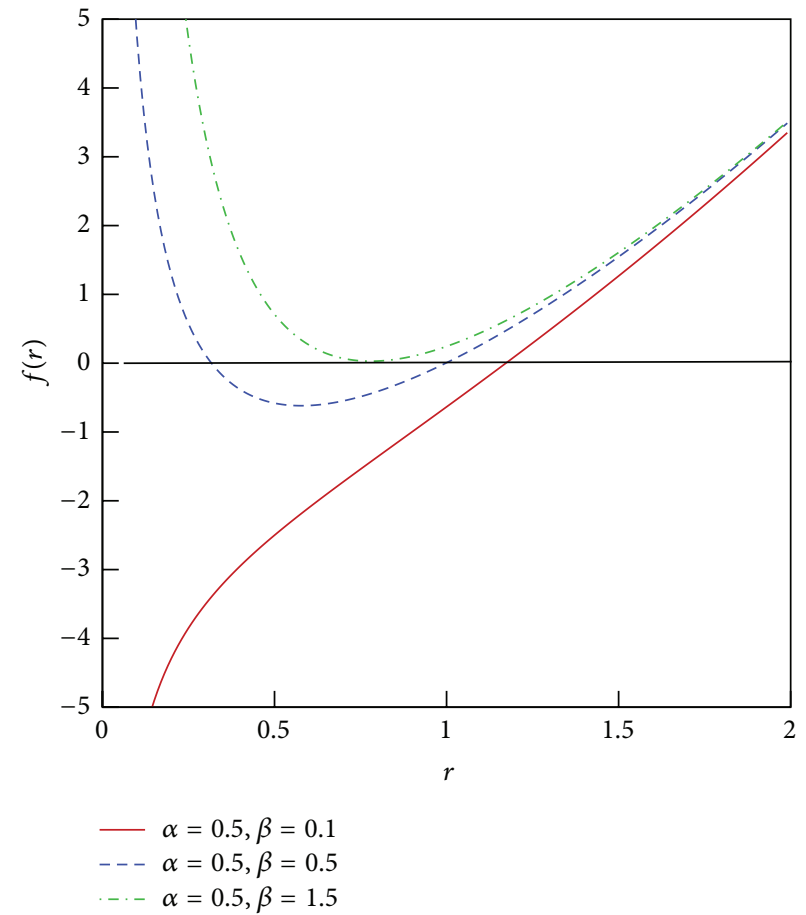

(b)

Figure 2: (a) shows the evolution of $f(r)$ versus $r$ for $q=1$ and $m=2.5$. In (b) the behavior of $f(r)$ is illustrated for $m=3.5$ and $q=1.3$. Here we have taken $l=b=1$ and $\Xi=1.25$.

depending on the metric parameters. We also see that for fixed value of the other parameters, the number of horizons decreases with increasing $\alpha$ or $\beta$. An interesting behavior occurred for small $\beta$. Indeed, for $\beta<\beta_{c}$, the black string coupled to ENd field has a nonextreme horizon in which the metric function may be negative near the origin for $\beta<\beta_{c}$, as it happens for Schwarzschild black hole. This is in contrast to the black string solutions of linear Maxwell theory. See the right panel of Figure 2.

\section{Thermodynamics of Dilaton Black String}

In this section we want to obtain conserved and thermodynamic quantities of the charged rotating dilaton black string. In order to obtain the conserved charges of the spacetime we use the counterterm method [64-66] inspired by (A)dS/CFT correspondence. We find the finite stress-energy tensor which removes the divergences of the action in the presence of dilaton field as

$$
T^{a b}=\frac{1}{8 \pi}\left[\Theta^{a b}-\Theta h^{a b}+\frac{2}{l_{\mathrm{eff}}} h^{a b}\right]
$$

where $l_{\text {eff }}$ is given by

$$
l_{\text {eff }}^{2}=\frac{\left(\alpha^{2}-3\right)}{\Lambda} e^{-2 \alpha \Phi} .
$$

It is important to note that the counterterm in (33) has the same form as in the case of asymptotically AdS solutions with zero curvature boundary, where $l$ is replaced by $l_{\text {eff. }}$. In particular, in the absence of the dilaton field $(\alpha=0)$, we have $l_{\text {eff }}^{2} \rightarrow l^{2}=-3 / \Lambda$ for the AdS spacetimes.

We choose the Killing vector field $\xi$ on spacelike surface $\mathscr{B}$ in $\partial \mathscr{M}$ with metric $\sigma_{i j}$. Then, the quasilocal conserved quantities may be obtained from the following relation [41]:

$$
Q(\xi)=\int_{\mathscr{B}} d^{2} x \sqrt{\sigma} T_{a b} n^{a} \xi^{b},
$$

where $\sigma$ is the determinant of the boundary metric $\sigma_{i j}$ and $n^{a}$ is the unit normal vector on the boundary $\mathscr{B}$. In our case, the boundary $\mathscr{B}$ has two Killing vector fields timelike $(\partial / \partial t)$ and rotational $(\partial / \partial \varphi)$. The corresponding conserved charges are the quasilocal mass and angular momentum. It is a matter of calculation to show that the mass and angular momentum per unit length of the rotating dilaton string, when the boundary $\mathscr{B}$ goes to infinity, can be calculated as

$$
\begin{gathered}
M=\frac{b^{\gamma}}{8 l}\left(\frac{\left(3-\alpha^{2}\right) \Xi^{2}+\alpha^{2}-1}{1+\alpha^{2}}\right) m, \\
J=\frac{b^{\gamma}}{8 l}\left(\frac{3-\alpha^{2}}{1+\alpha^{2}}\right) \Xi m a .
\end{gathered}
$$

When $a=0(\Xi=1)$, the angular momentum per unit length vanishes, and therefore $a$ is the rotational parameters of the spacetime.

Next, we calculate the entropy of the dilaton black string. Since the area law of entropy is universal and applies to all 

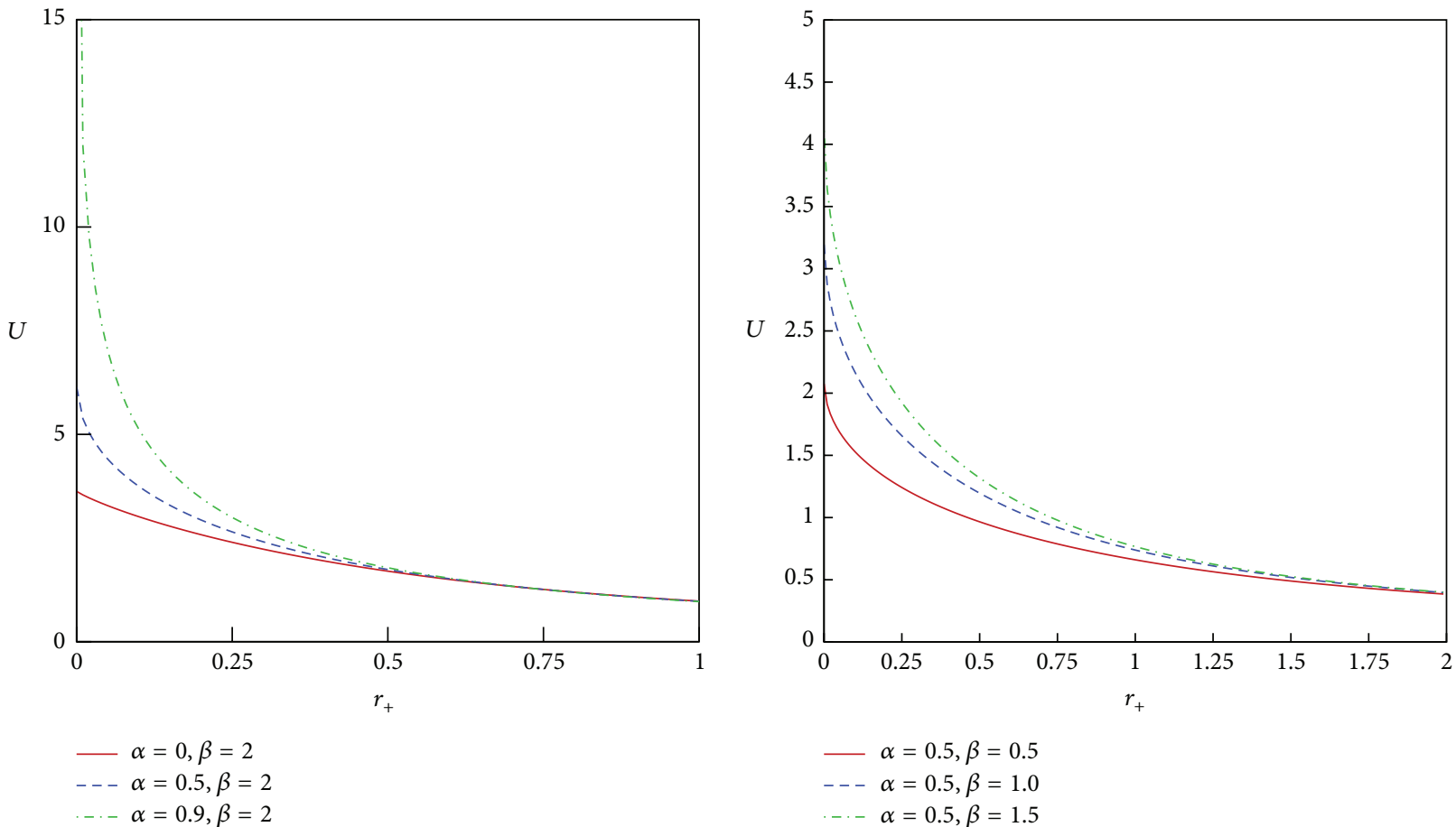

Figure 3: The evolution of $U$ versus $r_{+}$for $q=1, b=1, \Xi=1.25$, and different $\beta$ and $\alpha$.

kinds of black holes and black strings in Einstein gravity, the entropy per unit length of the string is obtained as

$$
S=\frac{\pi \Xi b^{\gamma} r_{+}^{2-\gamma}}{2 l}
$$

Using Gauss' law, we calculate the flux of the electromagnetic field at infinity to obtain the electric charge per unit length of black string. We find

$$
Q=\frac{\Xi q}{2 l}
$$

Also, the electric potential $U$, measured at infinity with respect to the horizon $r_{+}$, is defined by $[67,68]$

$$
U=\left.A_{\mu} \chi^{\mu}\right|_{r \rightarrow \infty}-\left.A_{\mu} \chi^{\mu}\right|_{r=r_{+}},
$$

where $\chi=\partial_{t}+\Omega \partial_{\phi}$ is the null generator of the event horizon. One can easily show that the vector potential $A_{\mu}$ corresponding to the electromagnetic tensors (12) and (13) can be written as

$$
\begin{aligned}
A_{\mu}= & b^{\gamma} \beta\left(\alpha^{2}+1\right)\left(\frac{\beta b^{\gamma}}{q}\right)^{(1-\gamma) /(\gamma-2)} \\
& \times\left(\frac{1-\alpha^{2}}{4}\right)^{1 /(2 \gamma-4)} \Upsilon(r)\left(\Xi \delta_{\mu}^{t}-a \delta_{\mu}^{\phi}\right),
\end{aligned}
$$

where

$$
\begin{gathered}
\Upsilon(r)=-\frac{1}{4} \Gamma\left(\frac{\alpha^{2}+1}{4}, \frac{1-\alpha^{2}}{4} L_{W}(\eta)\right) \\
+\frac{1}{\alpha^{2}-1}\left\{\Gamma\left(\frac{\alpha^{2}+5}{4}, \frac{1-\alpha^{2}}{4} L_{W}(\eta)\right)\right. \\
\left.-\frac{1}{2} \Gamma\left(\frac{\alpha^{2}+1}{4}\right)\right\} .
\end{gathered}
$$

Therefore, the electric potential is obtained as

$$
\begin{aligned}
U= & \frac{b^{\gamma} \beta}{\Xi}\left(\alpha^{2}+1\right)\left(\frac{\beta b^{\gamma}}{q}\right)^{(1-\gamma) /(\gamma-2)} \\
& \times\left(\frac{1-\alpha^{2}}{4}\right)^{1 /(2 \gamma-4)} \Upsilon\left(r_{+}\right) .
\end{aligned}
$$

Expanding for large value of $\beta$, we get

$$
U=\frac{q}{\Xi r_{+}}-\frac{b^{-2 \gamma}\left(\alpha^{2}+1\right)}{2 \beta^{2}\left(\alpha^{2}+5\right)} \frac{q^{3}}{\Xi r_{+}^{5-2 \gamma}}+O\left(\frac{1}{\beta^{4}}\right),
$$

which indicates that for $\beta \rightarrow \infty$, our expression reduces to the electric potential of black string in EMd gravity [41]. The behaviors of the electric potential $U$ as a function of horizon radius $r_{+}$are plotted in Figure 3. These figures show that, in contrast to the electric potential of black string in EMd theory [41], here the electric potential has a finite value as $r_{+} \rightarrow 0$. This is due to the nonlinear nature of electrodynamic field. 
Now, we are in a position to verify the first law of thermodynamics. In order to do this, we obtain mass $M$ as a function of extensive quantities $S, J$, and $Q$. Using the expressions for the mass, the angular momenta, the entropy, and the charge given in (36), (37), and (38), and the fact that $f\left(r_{+}\right)=0$, one can obtain a Smarr-type formula as

$$
M(S, J, Q)=\frac{\left[\left(3-\alpha^{2}\right) Z+\alpha^{2}-1\right] J}{\left(3-\alpha^{2}\right) \sqrt{Z(Z-1)}}
$$

where $Z=\Xi^{2}$ is the positive real root of the following equation:

$$
\begin{aligned}
& 2 l^{2} \pi J\left(\frac{2 l S}{\pi \sqrt{Z}}\right)^{\left(\alpha^{2}-1\right) / 2}+\frac{l^{2} \pi b^{\alpha^{2}}}{4} \beta Q \sqrt{Z-1} \\
& \times\left(\frac{1-\alpha^{2}}{4}\right)^{\left(3-\alpha^{2}\right) / 4}\left(\frac{\beta S}{\pi Q}\right)^{\left(\alpha^{2}-1\right) / 2}\left(\alpha^{2}-3\right)\left(\alpha^{2}+1\right) \\
& \times\left\{\Gamma\left(\frac{\alpha^{2}-3}{4}, \frac{1-\alpha^{2}}{4} L_{W}(\zeta)\right)-\Gamma\left(\frac{\alpha^{2}-3}{4}\right)\right. \\
& \quad-\frac{16}{\left(\alpha^{2}-1\right)^{2}}\left[\Gamma\left(\frac{\alpha^{2}+5}{4}, \frac{1-\alpha^{2}}{4} L_{W}(\zeta)\right)\right. \\
& +b^{\alpha^{2}} S\left(\beta^{2} l^{2}-\frac{3}{2}\right)\left(\alpha^{2}+1\right) \sqrt{Z-1}=0,
\end{aligned}
$$

where $\zeta=\pi^{2} Q^{2} / S^{2} \beta^{2}$. Now, if we consider the parameters $S$, $J$, and $Q$ as a complete set of extensive parameters for the mass $M(S, J, Q)$ and define the intensive parameters conjugate to $S$, $J$, and $Q$ as

$$
T=\left(\frac{\partial M}{\partial S}\right)_{J, Q}, \quad \Omega=\left(\frac{\partial M}{\partial J}\right)_{S, Q}, \quad U=\left(\frac{\partial M}{\partial Q}\right)_{S, J},
$$

then we can numerically check that the intensive quantities calculated by (46) coincide with (28), (29), and (42). This implies that the first law of thermodynamics is satisfied on the horizon of the rotating dilaton black string; namely,

$$
d M=T d S+\Omega d J+U d Q
$$

\section{Conclusions}

We constructed a new class of four-dimensional rotating black string solutions with cylindrical or toroidal horizons coupled to EN electrodynamic and dilaton fields. The presence of the Liouville-type dilaton potential, which is regarded as the generalization of the cosmological constant, changes the asymptotic behavior of the solutions to be neither flat nor (A)dS [33-35]. The obtained metric function $f(r)$ in (22) has a complicated form due to the presence of both dilaton and EN electrodynamics in the action. However, we have studied its physical properties and in particular the casual structure of the solutions. We found that when the nonlinear parameter goes to infinity, $\beta \rightarrow \infty$, our solutions reduce to charged rotating dilaton black string of EMd gravity [41]. While in the absence of the dilaton field, $(\alpha=0=\gamma)$, they restore charged rotating black string coupled to $\mathrm{EN}$ electrodynamics [5]. Interestingly enough, we found that the electric potential has a finite value as $r_{+} \rightarrow 0$ and goes to zero as $r_{+} \rightarrow \infty$. This is in contrast to the electric field of charged rotating black string of EMd gravity [41] and originates from the nonlinear nature of the electrodynamic field. We present the suitable counterterm which removes the divergences of the action in the presence of a Liouville-type dilaton potential. We calculated temperature, mass, angular momentum, entropy, charge, and electric potential of the rotating dilaton black string. We find a Smarr-type formula for the mass, $M=M(S, J, Q)$, and verified numerically that the first law of thermodynamics is satisfied on the horizon.

The generalization of the present dilaton rotating string, with one rotation parameter, to all higher dimensions with complete set of rotation parameters, and also the case of magnetic dilaton string/brane supported by EN electrodynamics are now under investigation and will appear in our future works.

\section{Conflict of Interests}

The author declares that there is no conflict of interests regarding the publication of this paper.

\section{Acknowledgments}

The author thanks Shiraz University Research Council. This work has been supported financially by Research Institute for Astronomy and Astrophysics of Maragha (RIAAM), Iran.

\section{References}

[1] M. Born and L. Infeld, "Foundations of the new field theory," Proceedings of the Royal Society A, vol. 144, no. 852, pp. 425-451, 1934.

[2] H. H. Soleng, "Charged black points in general relativity coupled to the logarithmic U(1) gauge theory," Physical Review $D$, vol. 52, no. 10, pp. 6178-6181, 1995.

[3] S. H. Hendi, "Asymptotic charged BTZ black hole solutions," Journal of High Energy Physics, vol. 2012, article 065, 2012.

[4] S. H. Hendi, "Asymptotic Reissner-Nordström black holes," Annals of Physics, vol. 333, pp. 282-289, 2013.

[5] S. H. Hendi and A. Sheykhi, "Charged rotating black string in gravitating nonlinear electromagnetic fields," Physical Review $D$, vol. 88, no. 4, Article ID 044044, 7 pages, 2013.

[6] E. Ayon-Beato and A. Garcia, "Regular black hole in general relativity coupled to nonlinear electrodynamics," Physical Review Letters, vol. 80, p. 5056, 1998.

[7] E. S. Fradkin and A. A. Tseytlin, "Non-linear electrodynamics from quantized strings," Physics Letters. B, vol. 163, no. 1-4, pp. 123-130, 1985. 
[8] R. R. Metsaev, M. A. Rahmanov, and A. A. Tseytlin, "The BornInfeld action as the effective action in the open superstring theory," Physics Letters B, vol. 193, no. 2-3, pp. 207-212, 1987.

[9] E. Bergshoeff, E. Sezgin, C. N. Pope, and P. K. Townsend, "The Born-Infeld action from conformal invariance of the open superstring," Physics Letters B, vol. 188, no. 1, pp. 70-74, 1987.

[10] A. A. Tseytlin, "On non-abelian generalisation of the BornInfeld action in string theory," Nuclear Physics B, vol. 501, no. 1, pp. 41-52, 1997.

[11] D. Brecher and M. J. Perry, "Bound states of D-branes and the non-abelian Born-Infeld action," Nuclear Physics. B, vol. 527, no. 1-2, pp. 121-141, 1998.

[12] C. G. Callan, C. Lovelace, C. R. Nappi, and S. A. Yost, "Loop corrections to superstring equations of motion," Nuclear Physics B, vol. 308, no. 2-3, pp. 221-284, 1988.

[13] O. D. Andreev and A. A. Tseytlin, "Partition-function representation for the open superstring effective action: cancellation of Möbius infinities and derivative corrections to Born-Infeld Lagrangian," Nuclear Physics B, vol. 311, no. 1, pp. 205-252, 1988/89.

[14] R. Leigh, "Dirac-Born-Infeld action from Dirichlet $\sigma$-model," Modern Physics Letters A, vol. 4, no. 28, pp. 2767-2772, 1989.

[15] G. Boillat, "Nonlinear electrodynamics: lagrangians and equations of motion," Journal of Mathematical Physics, vol. 11, no. 3 , pp. 941-951, 1970.

[16] G. Boillat, "Simple waves in N-dimensional propagation," Journal of Mathematical Physics, vol. 11, no. 4, pp. 1482-1483, 1970.

[17] G. W. Gibbons and D. A. Rasheed, "Electric-magnetic duality rotations in non-linear electrodynamics," Nuclear Physics B, vol. 454, no. 1-2, pp. 185-206, 1995.

[18] M. Brigante, H. Liu, R. C. Myers, S. Shenker, and S. Yaida, "Viscosity bound violation in higher derivative gravity," Physical Review D, vol. 77, no. 12, Article ID 126006, 2008.

[19] Y. Kats and P. Petrov, "Effect of curvature squared corrections in AdS on the viscosity of the dual gauge theory," Journal of High Energy Physics, vol. 2009, no. 1, article 044, 2009.

[20] P. Kovtun, D. T. Son, and A. O. Starinets, "Holography and hydrodynamics: diffusion on stretched horizons," Journal of High Energy Physics, vol. 10, article 064, 2003.

[21] R. G. Cai and Y.-W. Sun, "Shear viscosity from AdS Born-Infeld black holes," Journal of High Energy Physics, vol. 9, p. 115, 2008.

[22] J. Jing and S. Chen, "Quasinormal modes of a black hole in the deformed Hořava-Lifshitz gravity," Physics Letters B, vol. 687, no. 2-3, pp. 124-128, 2010.

[23] R. Gregory, S. Kanno, and J. Soda, "Holographic superconductors with higher curvature corrections," Journal of High Energy Physics, vol. 10, article 010, 2009.

[24] H. J. Mosquera Cuesta and J. M. Salim, "Non-linear electrodynamics and the gravitational redshift of highly magnetized neutron stars," Monthly Notices of the Royal Astronomical Society, vol. 354, pp. L55-L59, 2004.

[25] H. J. M. Cuesta and J. M. Salim, "Nonlinear electrodynamics and the surface redshift of pulsars," The Astrophysical Journal Letters, vol. 608, no. 2, pp. 925-929, 2004.

[26] G. W. Gibbons and K. Maeda, "Black holes and membranes in higher-dimensional theories with dilaton fields," Nuclear Physics B, vol. 298, no. 4, pp. 741-775, 1988.

[27] T. Koikawa and M. Yoshimura, "Dilaton fields and event horizon," Physics Letters B, vol. 189, no. 1-2, pp. 29-33, 1987.

[28] D. Brill and J. Horowitz, "Negative energy in string theory," Physics Letters B, vol. 262, no. 4, pp. 437-443, 1991.
[29] D. Garfinkle, G. T. Horowitz, and A. Strominger, "Charged black holes in string theory," Physical Review D, vol. 43, no. 10, pp. 3140-3143, 1991.

[30] R. Gregory and J. A. Harvey, "Black holes with a massive dilaton," Physical Review D, vol. 47, no. 6, pp. 2411-2422, 1993.

[31] M. Rakhmanov, "Dilaton black holes with electric charge," Physical Review D, vol. 50, no. 8, pp. 5155-5163, 1994.

[32] G. T. Horowitz and A. Strominger, "Black strings and p-branes," Nuclear Physics B, vol. 360, no. 1, pp. 197-209, 1991.

[33] S. J. Poletti and D. L. Wiltshire, "Global properties of static spherically symmetric charged dilaton spacetimes with a Liouville potential," Physical Review D, vol. 50, no. 12, pp. 7260-7270, 1994.

[34] S. J. Poletti, J. Twamley, and D. L. Wiltshire, "Charged dilaton black holes with a cosmological constant," Physical Review D, vol. 51, no. 10, pp. 5720-5724, 1995.

[35] S. Mignemi and D. L. Wiltshire, "Black holes in higherderivative gravity theories," Physical Review D, vol. 46, no. 4, pp. 1475-1506, 1992.

[36] K. C. Chan, J. H. Horne, and R. B. Mann, "Charged dilaton black holes with unusual asymptotics," Nuclear Physics B, vol. 447, no. 2-3, pp. 441-461, 1995.

[37] R.-G. Cai, J.-Y. Ji, and K.-S. Soh, “Topological dilaton black holes," Physical Review D, vol. 57, no. 10, pp. 6547-6550, 1998.

[38] R.-G. Cai and Y.-Z. Zhang, "Holography and brane cosmology in domain wall backgrounds," Physical Review D, vol. 64, no. 10, Article ID 104015, 8 pages, 2001.

[39] G. Clement, D. Gal'tsov, and C. Leygnac, "Linear dilaton black holes," Physical Review D, vol. 67, no. 2, Article ID 024012, 2003.

[40] G. Clément and C. Leygnac, "Non-asymptotically flat, non-AdS dilaton black holes," Physical Review D, vol. 70, no. 8, Article ID 084018, 2004.

[41] M. H. Dehghani and N. Farhangkhah, "Charged rotating dilaton black strings," Physical Review D, vol. 71, no. 4, Article ID 044008, 7 pages, 2005.

[42] M. H. Dehghani, "Magnetic strings in dilaton gravity," Physical Review D, vol. 71, Article ID 064010, 2005.

[43] A. Sheykhi, M. H. Dehghani, N. Riazi, and J. Pakravan, "Thermodynamics of rotating solutions in $(n+1)$-dimensional Einstein-Maxwell-dilaton gravity," Physical Review D, vol. 74, Article ID 084016, 2006.

[44] A. Sheykhi, M. H. Dehghani, and N. Riazi, "Magnetic branes in $(n+1)$-dimensional Einstein-Maxwell-dilaton gravity," Physical Review D, vol. 75, no. 4, Article ID 044020, 2007.

[45] A. Sheykhi, "Thermodynamics of charged topological dilaton black holes," Physical Review D, vol. 76, no. 12, Article ID 124025, 2007.

[46] R. Yamazaki and D. Ida, "Black holes in three-dimensional Einstein-Born-Infeld-dilaton theory," Physical Review D, vol. 64, no. 2, Article ID 024009, 6 pages, 2001.

[47] S. S. Yazadjiev, "Einstein-Born-Infeld-dilaton black holes in nonasymptotically flat spacetimes," Physical Review D, vol. 72, no. 4, Article ID 044006, 6 pages, 2005.

[48] A. Sheykhi, N. Riazi, and M. H. Mahzoon, "Asymptotically nonflat Einstein-Born-Infeld-dilaton black holes with Liouvilletype potential," Physical Review D, vol. 74, no. 4, Article ID 044025, 2006.

[49] T. Tamaki and T. Torii, "Gravitating BIon and BIon black hole with a dilaton," Physical Review D, vol. 62, no. 6, Article ID 061501, 5 pages, 2000. 
[50] G. Clément and D. Gal'tsov, "Solitons and black holes in Einstein-Born-Infeld-Dilaton theory," Physical Review D, vol. 62, no. 12, Article ID 124013, 10 pages, 2000.

[51] S. S. Yazadjiev, P. P. Fiziev, T. L. Boyadjiev, and M. D. Todorov, "Electrically charged einstein-born-infeld black holes with massive dilaton," Modern Physics Letters A, vol. 16, no. 33, pp. 21432149, 2001.

[52] A. Sheykhi, "Topological Born-Infeld-dilaton black holes," Physics Letters B, vol. 662, pp. 7-13, 2008.

[53] M. H. Dehghani, S. H. Hendi, A. Sheykhi, and H. Rastegar Sedehi, "Thermodynamics of rotating black branes in Einstein-Born-Infeld-dilaton gravity," Journal of Cosmology and Astroparticle Physics, vol. 2, article 020, 2007.

[54] A. Sheykhi and N. Riazi, "Thermodynamics of black holes in $(n+1)$-dimensional Einstein-Born-Infeld-dilaton gravity," Physical Review D, vol. 75, no. 2, Article ID 024021, 2007.

[55] A. Sheykhi, "Thermodynamical properties of topological BornInfeld-dilaton black holes," International Journal of Modern Physics D, vol. 18, no. 1, pp. 25-42, 2009.

[56] M. H. Dehghani, A. Sheykhi, and S. H. Hendi, "Magnetic strings in Einstein-Born-Infeld-dilaton gravity," Physics Letters B, vol. 659, no. 3, pp. 476-482, 2008.

[57] I. Z. Stefanov, S. S. Yazadjiev, and M. D. Todorov, "Scalar-tensor black holes coupled to Born-Infeld nonlinear electrodynamics," Physical Review D, vol. 75, no. 8, Article ID 084036, 2007.

[58] I. Z. Stefanov, S. S. Yazadjiev, and M. D. Todorov, "Scalartensor black holes coupled to Euler-Heisenberg nonlinear electrodynamics," Modern Physics Letters A: Particles and Fields, Gravitation, Cosmology, Nuclear Physics, vol. 22, no. 17, pp. 12171231, 2007.

[59] A. Sheykhi and S. Hajkhalili, Physical Review D. In press.

[60] J. P. Lemos, "Two-dimensional black holes and planar general relativity," Classical and Quantum Gravity, vol. 12, no. 4, pp. 1081-1086, 1995.

[61] J. P. Lemos, "Three-dimensional black holes and cylindrical general relativity," Physics Letters B, vol. 353, no. 1, pp. 46-51, 1995.

[62] M. Abramowitz and I. A. Stegun, Handbook of Mathematical Functions, Dover, New York, NY, USA, 1972.

[63] R. M. Corless, "On the LambertW function," Advances in Computational Mathematics, vol. 5, pp. 329-359, 1996.

[64] J. D. Brown and J. W. York, "Quasilocal energy and conserved charges derived from the gravitational action," Physical Review D, vol. 47, p. 1407, 1993.

[65] J. Maldacena, "The large $N$ limit of superconformal field theories and supergravity," Advances in Theoretical and Mathematical Physics, vol. 2, no. 2, pp. 231-252, 1998.

[66] E. Witten, "Anti de Sitter space and holography," Advances in Theoretical and Mathematical Physics, vol. 2, no. 2, pp. 253-291, 1998.

[67] M. Cvetic and S. S. Gubser, "Phases of R-charged black holes, spinning branes and strongly coupled gauge theories," Journal of High Energy Physic, vol. 1999, no. 04, article 024, 1999.

[68] M. M. Caldarelli, G. Cognola, and D. Klemm, "Thermodynamics of Kerr-Newman-AdS black holes and conformal field theories," Classical and Quantum Gravity, vol. 17, no. 2, pp. 399420, 2000. 

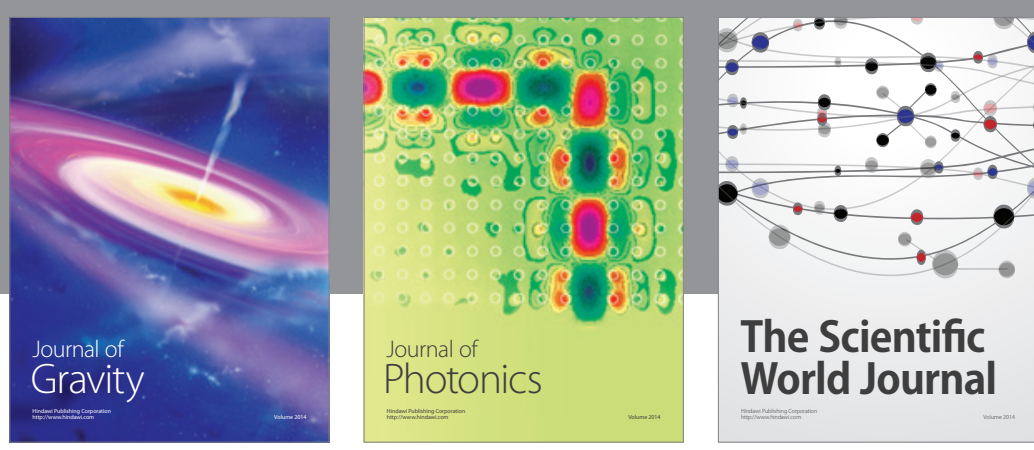

The Scientific World Journal
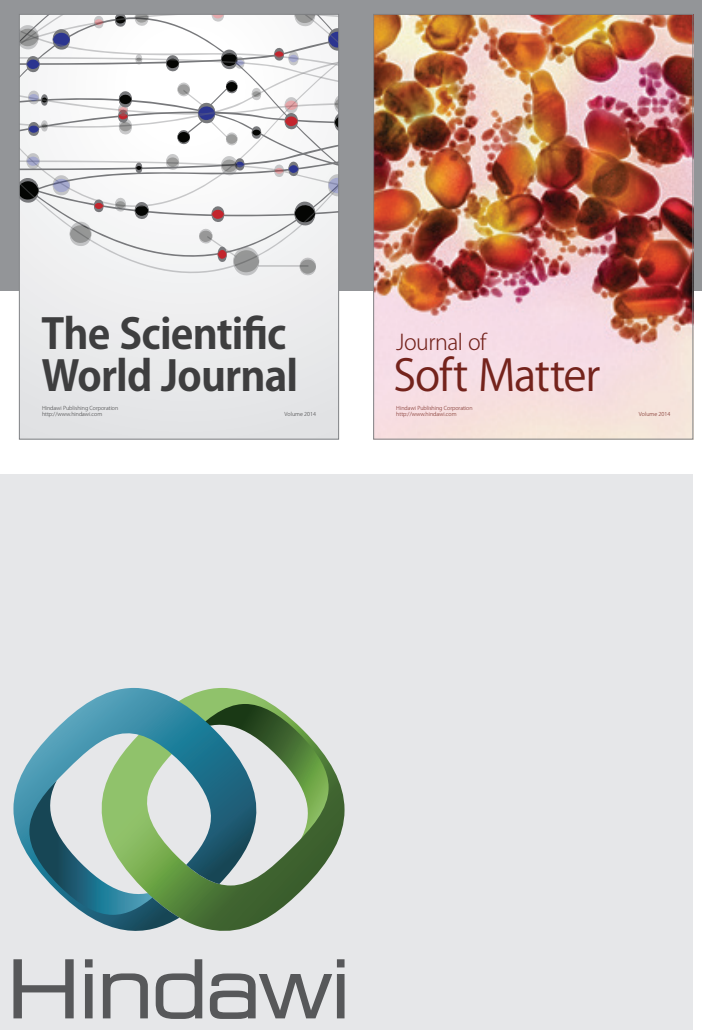

Submit your manuscripts at

http://www.hindawi.com

nternational Journal of

Statistical Mechanics
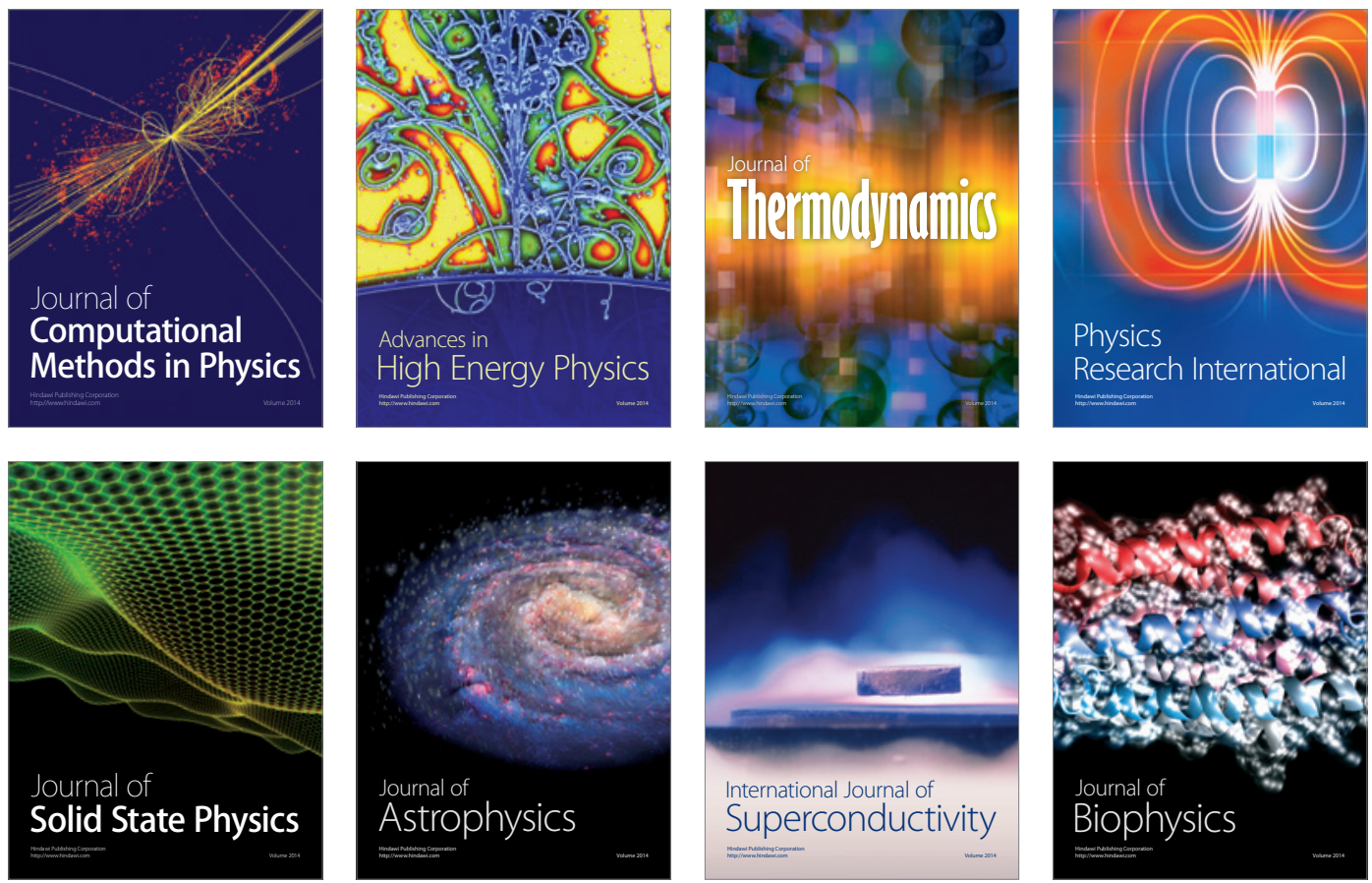
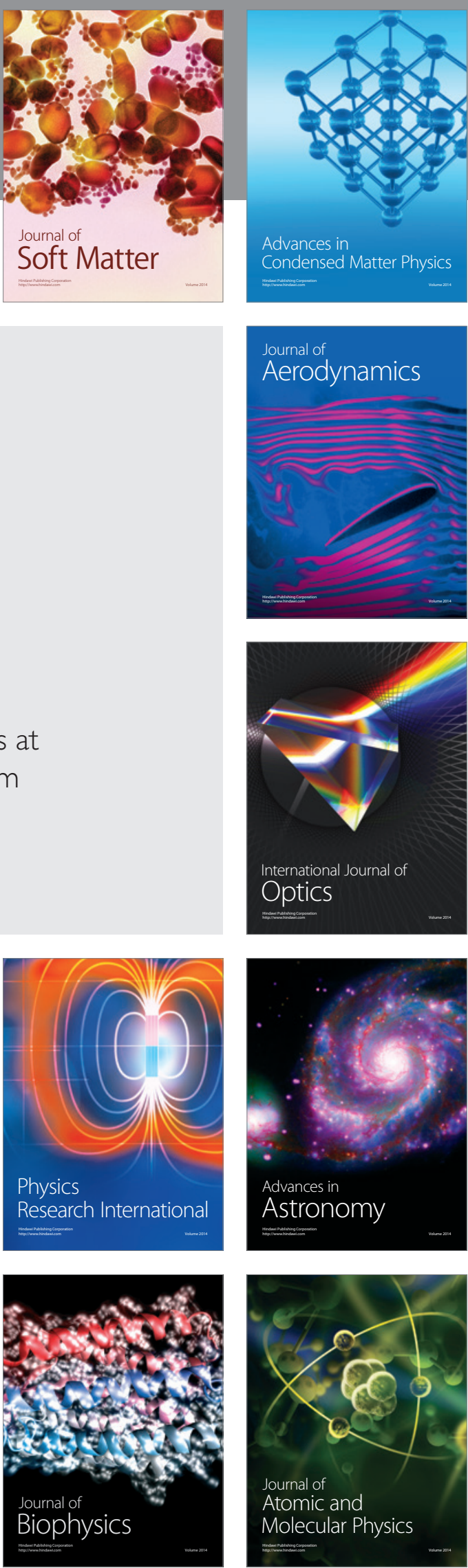